\title{
Eliminating Broadcast Storming in Vehicular Ad-Hoc Networks
}

\author{
Umar Hayat \\ Department of Computer Science \\ and IT \\ Lahore Leads University \\ Lahore, Pakistan
}

\author{
Razi Iqbal \\ College of Computer and \\ Information Technology \\ American University in the Emirates \\ Dubai, UAE
}

\author{
Jamal Diab \\ College of Computer and \\ Information Technology \\ American University in the Emirates \\ Dubai, UAE
}

\begin{abstract}
VANETs (Vehicular Ad-hoc Networks) offer diversity of appealing applications. A lot of applications offered by VANETs depend upon the propagation of messages from one vehicle to another vehicle in the network. Several algorithms for useful broadcasting of safety/ warning messages in the network have been presented by different researchers. Vehicles on roads are increasing day by day. Due to this increased number of vehicles and especially during the crest hours, when the networks become very dense, dissemination of the messages blindly in the network causes problems like packet collisions, data thrashing and broadcast storming. In this research, a relative speed based waiting time algorithm has been presented for avoiding broadcast storming problem in the VANETS especially in dense environment. This proposed algorithm calculates the waiting time for each vehicle after receiving the safety/ warning messages according to the relative speed of the vehicles, the distance between the vehicles and range of vehicles. The results show that the proposed relative speed based algorithm is better than already existing algorithms like blind flooding and dynamically broadcasting waiting time algorithm which uses number of neighbors and distance between the vehicles for calculating the waiting time.
\end{abstract}

Keywords-VANETs; Intelligent Transportation Systems; Broadcast Storming; Distance based flooding

\section{INTRODUCTION}

VANETs are Ad-hoc networks for vehicles on roads. These networks are temporary networks created for small amount of time since vehicles are moving at a rapid speed. Normally these networks are used to provide safety on road by transmitted information from other vehicles or infrastructure. VANETs fall under the broader category of ITS (Intelligent Transportation Systems). ITS are used for improving the efficiency of transportation systems using advanced technologies. The major objectives of ITS are to enhance road safety, provide convenience of information, reducing traffic congestions, improving user experience on roads and enforcing traffic laws [1]. Car navigation systems, railroad crossing alarms, speed checking cameras and electronic tolls gates are all examples of ITS.

In VANETs information transmission between vehicles should be reliable enough to ensure maximum safety on roads. This information transmission can be between 2 vehicles or between several vehicles at the same time. This concept of sending information to several vehicles is called information broadcasting. In VANETs, many techniques like Simple
Flooding, Probabilistic Flooding, Neighbor knowledge Flooding, Area based Flooding, Distance based Flooding, and Location based Flooding [2][3] are available for broadcasting information between vehicles and infrastructures. Increase in broadcasting these messages can increase packet collision and data loss. This increase in packet collision and data loss due to dense network is called Broadcast Storming.

Several techniques are available to avoid broadcast storming in VANETS. Some of these techniques are 1persistence, slotted 1-persistence and weighted p-persistence techniques [4]. In order to minimize the broadcast storming problem, a new relative speed based waiting time calculation approach is adopted. This approach adjusts the waiting time of a vehicle for broadcasting a warning or safety message for other vehicles in the network dynamically with respect to the distance of the vehicle from the base vehicle and the relative speed. If the speed of the vehicle is slow, its waiting time is increased. The vehicle, which is moving with the highest speed and has more number of neighboring vehicles will have very little waiting time and will broadcast within no time.

In order to transmit information between vehicles a communication technology is required. However, since in VANETs, there are many vehicles on road and equipping all the vehicles with expensive modules would increase the overall cost of the system. There for the purpose of this research, a low cost and power efficient technology, ZigBee is used. ZigBee supports star, mesh and peer to peer topologies which are compatible with VANETs. It can provide high scalability and has better communication range as compared to Bluetooth and Wi-Fi devices. This technology can help in eliminating redundancy of safety messages being broadcasted, because it works in two different modes i.e. beacon mode and non-beacon mode that can facilitate the traffic in random directions. When the coordinator works with the help of batteries the beacon mode is used and in this way it proposes more power reduction and savings. On the other hand, nonbeacon is more preferred and gets more favor when coordinator is running on the main supply of power. Furthermore, some researches have shown that ZigBee works well with moving objects like vehicles even if traffic is congested. Moreover, since these wireless modules work in outdoor environment, it is important to use a technology which can sustain extreme weather conditions like snowfall and torridity. Different researches have shown that ZigBee performs well in extreme weather conditions. 
Rest of the paper is organized as follows: Section II deals with the related work in this field and analysis of already done research in this area, along with identifying the gaps for research. Section III proposes a model for eliminating the broadcast storming for four lanes scenario. Section IV explains the flow of the proposed system. Section V describes the proposed algorithm for eliminating the broadcast storming in VANETs. Finally the last section concludes the paper.

\section{RELATED WORK}

A lot of work has already been done to avoid the broadcast storming problem. This problem was firstly examined in the MANETs. Different authors have suggested different techniques to diminish the problem. These techniques are used to swiftly minimize the redundancy of the safety messages and are being used in VANETs for mitigation of the broadcast storming. This section describe literature review for the research.

NajafZadeh et al. [5] proposed a waiting time based algorithm named "Dynamic Broadcasting Algorithm" to reduce the number of rebroadcasts of same message time and again. In this method they describe that nodes prepare their neighbor's list by using hello beaconing. Their algorithm calculates the waiting time for a node to rebroadcast a message by number of neighbors and the distance between sender node and the other vehicles. By using simulation results, they have concluded that their presented dynamic broadcasting algorithm is better in performance than flooding technique and random waiting time protocol. This waiting time based algorithm helps to reduce the redundancy of safety messages and minimizes the broadcast storm.

Suriyapaibonwattana et al. [6] proposed an algorithm for minimizing broadcast storm in VANETs and called it "The Last One broadcasting method”. In this method, when a vehicle receives information, it rebroadcasts this information by using random probability. This method is very useful to minimize the broadcasting storm in VANETs but cannot fully eliminate the broadcast storm.

Chiasserini et al. [7] discussed a scheme for achieving a smart broadcast analogy by using a channel access mechanism in vehicular ad hoc networks systems. This scheme states that the vehicles, which are going to rebroadcast the warning messages, will access channel with the different priority. This priority will depend on the distance between the last vehicles, which rebroadcasted warning message from the vehicles which will rebroadcast.

Francisco J. Ros et al. [8] explained a protocol for overcoming broadcast storm in vehicular ad hoc networks. This is modified form of PBSM (Parameter less Broadcasting in Static Mobile Wireless Ad-hoc) and is called AckPBSM (Acknowledged PBSM). PBSM can work with both 2-hop and 1-hop topological information. In this method every vehicle use a buffer to store the alert message and this message is discarded after a certain time. This AckPBSM works same like PBSM except that in AckPBSM broadcast alert messages are acknowledged and due to this acknowledgement the vehicle which has already received the same message will not rebroadcast the same message. This phenomenon helps in reducing the broadcast storm.

Roberta Frachia et al. [9] proposed a broadcast storm avoiding algorithm which is based on distance between the vehicles. Their distance aware flooding technique depends on distance between the current node and closest neighboring node. This algorithm generates a waiting time for a message to rebroadcast. By using simulation results they have concluded that their proposed distance aware delayed flooding algorithm yields better performance to avoid broadcast storm in VANETs than other broadcast suppression techniques.

Koubek et al [10] proposed a scheme to minimize the number of vehicles, which broadcast the message regarding the identical event. Delays and redundancy of the data, which is being transmitted, have been removed significantly by using the proposed methodology. Due to erratic protocol used for broadcasting this ESSMD has decrementing probability for event response. This response rate has been enhanced by modified scheme, which is ESSMD + Rep. This scheme enhances the trustworthiness of the protocol by replicating broadcasts on base vehicles. ESSMD technique has been stimulated by the use of ideology of data aggregates, which is minimizing the redundancy of the transmission. This broadcast elimination technique seems to confine the base vehicles which are reporting on identical events in spite of cumulating information from numerous base vehicles at solitary point. This technique has been evaluated as much better technique than the already existing aggregation methods used for reducing the storm of broadcast messages. ESSMD + Rep uses the repetitions to enhance the consistency of booming broadcasts while the physical path is not burdened. By raising the repetitions enhanced reliability has been accomplished at charge of enhanced transmissions that can direct towards a flooded network. In both schemes i.e. Restricted-Mobility-Based protocol has been used in replacement of ordinary flooding protocols used for broadcasting.

These researches by the authors concerning the broadcast of safety/ warning messages between the vehicles on road has paid attention to the problems like medium contention, data packet collisions and broadcast storming etc. Their focus is mainly on medium access control, collision avoidance, message propagation protocols and broadcast storming avoidance algorithms and techniques. All these algorithms and techniques have benefits as well as their limitations. In this research, our focal point is the alleviation of broadcast storming in VANETs by using relative speed based technique to make the network efficient and reliable.

\section{PROPOSED SYSTEM MODEL}

This is a four lane road scenario. All of these vehicles are equipped with ZigBee devices as onboard equipment for communication with each other and with RSUs. In the given scenario, the vehicle " $A$ " collects the information of the accident first and starts to broadcast this information of the accident to other vehicles in the network. A message "ACCIDENT AHEAD" is transmitted to all vehicles in the communication range of vehicle " $A$ " and to the road side 
units. All these vehicles, which receive this message from vehicle " $A$ ", calculate their waiting time according to the proposed relative speed based technique. After calculating the waiting time, these vehicles start to count down and when this waiting time reaches to zero, they start broadcasting the received information to other vehicles in the network. If the waiting time of the vehicle has not reached to zero and meanwhile, this vehicle receives the same information from any other vehicle in the network or from RSU, then it does not broadcast and discards this information. Vehicles also broadcast the information received from RSUs. Like vehicle with onboard equipment, RSUs are able to receive and broadcast the messages in the network. These RSUs can also save the received information and can send this information to the vehicles, which later enter in the communication range of the RSU.

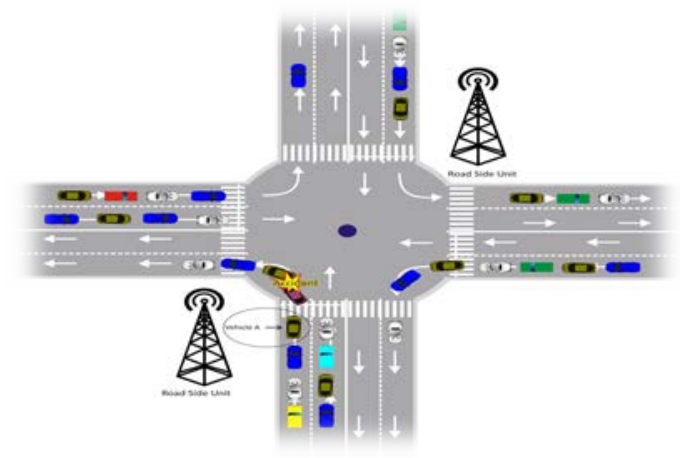

Fig. 1. Proposed system model

Fig. 2 shows a sparse network in which two vehicles are moving with the same speed. Due to moving with same speed, the relative speed of both the vehicles is zero. According to our proposed technique, when relative speed is zero, the wait time for rebroadcasting the warning/ safety message in the network becomes equal to maximum wait time for rebroadcasting. Due to this maximum wait time, the chances for receiving a duplicate packet of the same information are increased. As a result, rebroadcasting of the same message by both vehicles will not be ensured and hence broadcasting storm is minimized.

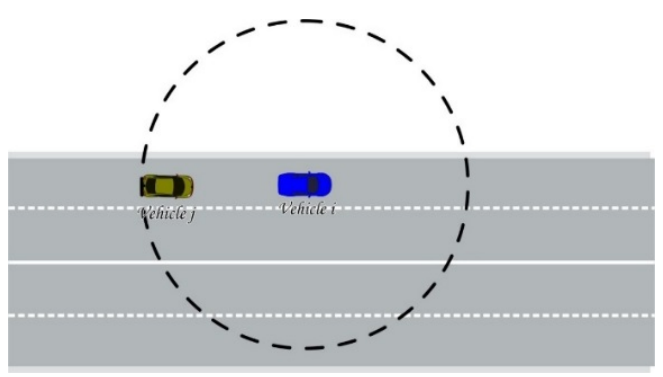

Fig. 2. A sparse network

Fig. 3 is a scenario of a traffic jam or traffic stuck on a signal. In this scenario, when all vehicles broadcast the warning/ safety messages according to the distance based technique or according to dynamic waiting time broadcasting algorithm, there are high chances of broadcast storming of the same information. In this scenario, almost every vehicle has some neighbors. Here, if we consider the relative speed of the vehicles for calculating waiting time for each vehicles to broadcast the safety/ warning message in the vehicular Ad-hoc network, it will be much beneficial than the other existing algorithms. In this case the speed of each vehicle within the network is approximately zero and hence their relative speed is also zero. Here the waiting time for each vehicle comes out equal to maximum waiting time required for broadcasting.

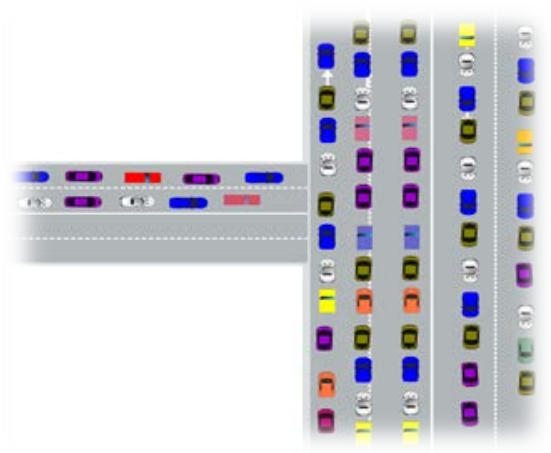

Fig. 3. Traffic Jam scenario

When each vehicle will wait for maximum time for rebroadcasting, there are more chances of getting the same information from the other vehicles. When a vehicle gets the same packet from other vehicle within the stipulated waiting time, it discards this packet rather than rebroadcasting. As a result of increased waiting time, a number of vehicles get the duplicate packet and discards it and hence broadcast storming problem is eliminated up to much extent as compared to other broadcasting techniques.

In Fig. 4, some vehicles in region " $A$ " are stopped on a signal and other vehicles in the network are moving with some speed. Vehicle "V1" receives the information and starts broadcasting this information to other vehicles in the network which are in its communication range. Other vehicles within the broadcasting range of the vehicle "V1" calculate their waiting time according to proposed relative speed based waiting time algorithm using their relative speed with respect to vehicle "V1". When vehicle "V2" counts down its waiting time to zero and no duplicate packet of same information is received from any other source in the network, it starts rebroadcasting the safety / warning messages received from vehicle "V1" or Road Side Units. Other vehicles in the communication range of vehicle "V2" calculate their waiting time according to proposed algorithm by using their relative speed with respect to "V2".

The vehicles with more difference in their speed have much relative speed and have less waiting time for broadcasting the safety/ warning message. Similarly the vehicles which are moving with same speed or have no speed i.e. stopped, their relative speed is approximately zero and they have more waiting time before broadcasting. As waiting time before broadcasting is increased, the number of vehicles broadcasting the warning/ safety message in the vehicular network is reduced. As number of vehicles broadcasting the warning / safety messages is reduced, the broadcast storming problem is automatically be minimized. 


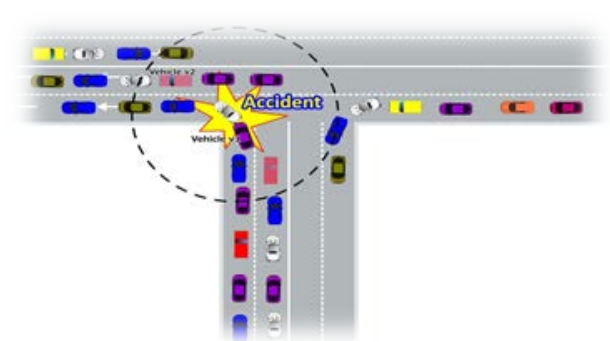

Fig. 4. Occurrence of an accident on traffic signal

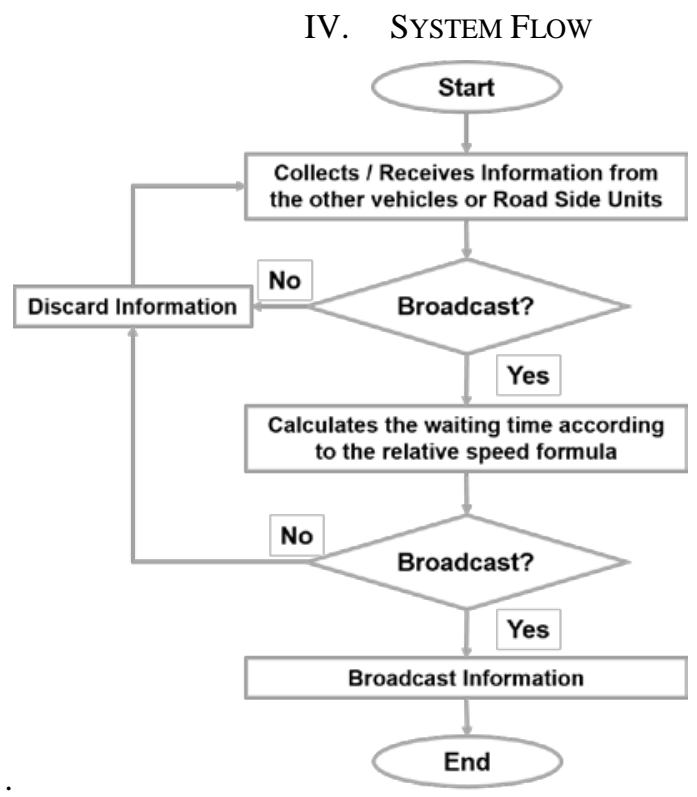

Fig. 5. Proposed System Flow of the model

The vehicle which collects information from environment or from Road Side Unit or any other source of the network, it checks whether it has received the same warning/ safety message already from any other source of the network. If it has already received the same packet it is discarded. If the vehicle has not already received the same packet, it calculates waiting time for broadcasting according to its relative speed with respect the sender vehicle. Now this vehicle waits until the waiting time counts down to zero. If the waiting time has not yet counts down to zero and in the meanwhile it receives the same information from any other vehicle in the network or from Road Side Unit, it discards the information and hence no rebroadcasting. If the waiting time has counts down to zero and the vehicle has not received the duplicate information from any source of the network it starts broadcasting the information in the network.

\section{Proposed Algorithm}

To tackle with the no broadcast and broadcast storming problems weighted p-persistence technique has been used. This technique works similar to p-persistence except that it uses probability for rebroadcasting the safety/ warning messages in the network which is calculates as under [11] :-

$$
P_{i j}=\frac{D_{i j}}{R}
$$

Where $D_{i j}$ refers to the distance between the sending and receiving vehicles and $\mathrm{R}$ is maximum range of network.

In 1-persistence, the network has multiple numbers of slots with the constraint that the vehicles should be within any one of the slots otherwise the vehicle will not be able to send and receive the information. In this technique, when a vehicle receives a message from any other vehicle in the network, it starts to wait for some specific time and when this time counts down to zero then this vehicle starts to broadcast the message. Each vehicle has different time for waiting. This difference in time for wait is due to the different slots to which the vehicles belong. In this technique, the vehicles calculate the time for wait as under [12]:-

$$
T_{S_{i j}}=S_{i j} * \mathrm{~T}
$$

Where $T$ represents the wait time which is required for the multiple hop transmission and $S_{i j}$ represents slots present within the broadcasting vehicle and receiving vehicle. We can calculate $S_{i j}$ as under:-

$$
S_{i j}=N_{s}\left(1-\left|\frac{\min \left(D_{i j}, R\right)}{R}\right|\right)
$$

Where $N_{s}$ represent total slots, $\mathrm{D}_{\mathrm{ij}}$ represents distance of broadcasting vehicle from the receiving vehicle and $\mathrm{R}$ is the range of the transmission. It is concluded that the vehicle which is at the most distance waits for less time.

A dynamic broadcasting technique has been proposed which is distance based technique, in which the base vehicle have knowledge of its neighboring vehicle with the help of hello beaconing. This technique considers not only the distance for calculating the wait time but also considers the number of neighboring vehicle. With the help of this technique the waiting time is calculated as under [13]:-

$$
W_{t}=\left(1-\frac{D_{i j}}{R}\right)^{n} W_{t_{\max }}
$$

Where $n$ represents the neighboring vehicles, $D_{i j}$ represents the distance of broadcasting vehicle form the receiving vehicle, $\mathrm{R}$ represents the range of the transmission and $W_{t_{\max }}$ represents the maximum wait time. If there is present only a single neighbor the vehicle does not wait and broadcasts the message. The vehicle which is at the most distance from the base vehicle and more vehicles are its neighboring vehicles then it will have high priority to broadcast. This technique has better performance than random waiting time but this technique suffers in dense networks like when the vehicles are stopped at traffic signal or traffic is jam, then in such scenarios almost every vehicle has a lot of neighboring vehicles. There will be again a broadcasting storm in the network. As we know that in the sparse vehicular network, there is no problem of broadcasting storm. Its most threat is present in the dense networks. In the dense networks the vehicles often have slow speed as compared to the sparse network. We have optimized the results by modifying the above dynamic broadcast technique by adding the relative speed of the vehicles to calculate the wait time for initiating the broadcasting. By our technique the waiting time for each vehicle present in the network is calculated as under:- 


$$
W_{t}=\left(\frac{R-S_{i j}}{R}\right)^{R S_{i j}} W_{t_{\max }}
$$

Where, $W_{t}$ represents the waiting time of vehicle for broadcasting the message, $\mathrm{R}$ represents the range of the forwarding/ broadcasting vehicles and $S_{i j}$ represents the distance between the vehicle "i" and vehicle “ $\mathrm{j}$ ", $\mathrm{RS}_{\mathrm{ij}}$ represents the relative speed of vehicle "i" with respect to vehicle " $\mathrm{j}$ " and $W_{t_{\max }}$ represents the maximum waiting time for a vehicle to wait before broadcasting the safety/ warning message in the network. With the addition of relative speed we have more optimized results for the reduction of the

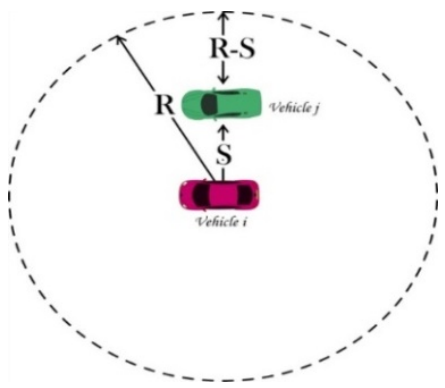

Fig. 6. Range of vehicle ' $i$ ' and its distance from vehicle 'j'

broadcasting storm in the dense networks than above dynamic technique and random time technique. The use of relative speed of the vehicles to alleviate the broadcasting storm in the VANETs is unprecedented.

Waiting time for vehicles, the time for which the vehicles has to wait before broadcasting has been calculated for both Dynamic Waiting Time Algorithm and our proposed Relative Speed Based Waiting Time Algorithm. Then the resulting waiting time of the both algorithms has been compared.

In the following table we have used the data of such road scenario where traffic is very dense and all the vehicles are moving with some speed. Some vehicles are moving with high speed and some are moving with low speed. Due to dense network, every vehicle has no. of neighbors and some relative speed with respect to other vehicles present in the network. Maximum waiting time has been chosen 20 milliseconds i.e. every vehicle will not wait more than 20 milliseconds before broadcasting if it would not get any duplicate information from any other source in the network.

The comparison of waiting time by using Dynamic Broadcasting Algorithm and proposed relative speed based waiting time algorithm shows that the waiting time for each vehicle by relative speed based algorithm is more or equal to the waiting time by dynamically broadcasting algorithm. It is clear that if each vehicle in the network has to wait for more, it has more chances of getting duplicate information from any other vehicle or road side unit in the network and has low probability for broadcasting the safety/ warning message in the network. The reduced probability for broadcasting results in less broadcasting nodes in the network as most of the vehicles receive duplicate packet of the information while waiting and discards this information instead of broadcasting. As a result, there will be few nodes broadcasting and forwarding the safety/ warning messages in the network. Table II shows the data and results for vehicles which are on a traffic signal, where some vehicles are moving with some speed and other vehicles are stopped on the signal and have zero relative speed. As it is a signal, there are more number of neighbors for each vehicle and by dynamic broadcasting algorithm waiting time will be less for each vehicle and these vehicle have more probability of broadcasting the safely/ warning messages in the network. But according to our proposed relative speed based algorithm, as it is traffic signal and relative speed of the vehicles is less or zero, each vehicle in the network has low probability of broadcasting the safety/ warning messages in the vehicular Ad-hoc network due to more waiting time.

TABLE I. EXPERIMENTAL DATA FOR RANDOMLy MOVING VEHICLES IN DENSE TRAFFic AREA

\begin{tabular}{|c|c|c|c|c|c|c|c|c|c|}
\hline $\mathbf{V}_{i}$ & $\mathbf{V}_{j}$ & $\begin{array}{l}\text { R } \\
\text { (m) }\end{array}$ & $\begin{array}{l}S_{i j} \\
(\mathbf{m})\end{array}$ & nn & $\begin{array}{l}\text { Relative } \\
\text { speed } \\
(\mathbf{k m} / \mathbf{h})\end{array}$ & $\begin{array}{l}W_{\text {tmax }} \\
\text { (ms) }\end{array}$ & $\begin{array}{l}\mathrm{W}_{t} \text { by Dynamic } \\
\text { waiting time } \\
\text { algo. } \\
\text { (ms) }\end{array}$ & $\begin{array}{l}\mathrm{W}_{t} \text { by our } \\
\text { proposed } \\
\text { waiting time } \\
\text { algo. } \\
\text { (ms) }\end{array}$ & $\begin{array}{l}\mathrm{W}_{t} \text { of relative speed } \\
\text { algorithm as compared } \\
\text { to dynamic waiting } \\
\text { time algorithm }\end{array}$ \\
\hline V1 & V2 & 100 & 50 & 10 & 5 & 20 & 0.195 & 0.625 & More \\
\hline V1 & V3 & 100 & 60 & 20 & 20 & 20 & $2.19 \times 10^{-7}$ & $2.19 \times 10^{-7}$ & Equal \\
\hline V1 & V4 & 100 & 45 & 25 & 15 & 20 & $6.45 \times 10-6$ & $2.45 \times 10-3$ & More \\
\hline V1 & V5 & 100 & 90 & 50 & 10 & 20 & 0 & $2.19 \times 10-9$ & More \\
\hline V2 & V3 & 100 & 80 & 20 & 15 & 20 & $2.97 \times 10-13$ & $6.55 \times 10-10$ & More \\
\hline V2 & V4 & 100 & 10 & 25 & 20 & 20 & 1.435 & 2.431 & More \\
\hline V3 & V4 & 100 & 100 & 25 & 35 & 20 & 0 & 0 & Equal \\
\hline V4 & V5 & 100 & 95 & 50 & 30 & 20 & $1.77 \times 10-14$ & $1.86 \times 10-8$ & More \\
\hline V3 & V5 & 100 & 75 & 50 & 20 & 20 & 0 & $1.82 \times 10-11$ & More \\
\hline V2 & V6 & 100 & 35 & 40 & 10 & 20 & $6.56 \times 10-7$ & 0.269 & More \\
\hline
\end{tabular}


TABLE II. EXPERIMENTAL DATA FOR TRAFFIC ON A SIGNAL

\begin{tabular}{|c|c|c|c|c|c|c|c|c|c|}
\hline $\mathbf{V}_{i}$ & $\mathbf{V}_{j}$ & $\begin{array}{l}R \\
(\mathrm{~m})\end{array}$ & $\begin{array}{l}S_{i j} \\
(\mathrm{~m})\end{array}$ & nn & $\begin{array}{l}\text { Relative } \\
\text { speed } \\
(\mathbf{k m} / \mathbf{h})\end{array}$ & $\begin{array}{l}\mathbf{W}_{\text {tmax }} \\
(\mathbf{m s})\end{array}$ & $\begin{array}{l}W_{t} \text { by Dynamic } \\
\text { waiting time } \\
\text { algo. } \\
\quad(\mathrm{ms})\end{array}$ & $\begin{array}{l}W_{t} \text { by our } \\
\text { proposed } \\
\text { waiting time } \\
\text { algo. } \\
\quad(\mathrm{ms})\end{array}$ & $\begin{array}{l}W_{t} \text { of relative speed } \\
\text { algorithm as compared } \\
\text { to dynamic waiting time } \\
\text { algorithm }\end{array}$ \\
\hline V1 & V2 & 100 & 2 & 15 & 5 & 20 & 14.771 & 18.078 & More \\
\hline V1 & V3 & 100 & 3 & 14 & 10 & 20 & 13.056 & 14.748 & More \\
\hline V1 & V4 & 100 & 10 & 13 & 10 & 20 & 5.083 & 6.970 & More \\
\hline V1 & V5 & 100 & 10 & 15 & 20 & 20 & 4.117 & 2.431 & Less \\
\hline V2 & V3 & 100 & 20 & 14 & 10 & 20 & 0.879 & 2.147 & More \\
\hline V2 & V4 & 100 & 15 & 13 & 0 & 20 & 2.419 & 20 & More \\
\hline V4 & V3 & 100 & 10 & 14 & 0 & 20 & 4.575 & 20 & More \\
\hline V3 & V4 & 100 & 15 & 13 & 0 & 20 & 2.419 & 20 & More \\
\hline V3 & V5 & 100 & 5 & 15 & 0 & 20 & 9.265 & 20 & More \\
\hline V4 & V5 & 100 & 4 & 15 & 0 & 20 & 10.841 & 20 & More \\
\hline
\end{tabular}

In Table III, the data for a traffic jam scenario has been used and the results have been calculated by both the algorithms. As it is traffic jam, the vehicles are stuck on the road. Each vehicle has more number of neighbors and due to traffic jam the network is highly dense and speed of each vehicle in the network is zero and hence the their relative speed is also zero. Due to more number of neighbors the Dynamically Broadcasting algorithm yields less waiting time for each vehicle as shown in the above table and have high probability of broadcasting the safety/ warning messages in the network. The proposed Relative Speed Based waiting time algorithm yields waiting time equal to maximum waiting time for each vehicle in the network due to zero relative speed. This means that each vehicle has to wait for maximum waiting time before broadcasting and has very low probability for broadcasting the warning/ safety messages in the network which results in less broadcasting nodes in the network.

TABLE III. EXPERIMENTAL DATA TRAFFIC JAM SCENARIO

\begin{tabular}{|c|c|c|c|c|c|c|c|c|c|}
\hline $\mathbf{V}_{\boldsymbol{i}}$ & $\mathbf{V}_{j}$ & $\begin{array}{l}R \\
\text { (m) }\end{array}$ & $\begin{array}{l}S_{i j} \\
(\mathrm{~m})\end{array}$ & nn & $\begin{array}{l}\text { Relative } \\
\text { speed } \\
(\mathbf{k m} / \mathbf{h})\end{array}$ & $\begin{array}{l}\mathbf{W}_{\text {tmax }} \\
(\mathrm{ms})\end{array}$ & $\begin{array}{l}W_{t} \text { by Dynamic } \\
\text { waiting time } \\
\text { algo. } \\
\quad(\mathrm{ms})\end{array}$ & $\begin{array}{l}W_{t} \text { by our } \\
\text { proposed } \\
\text { waiting time } \\
\text { algo. } \\
\quad(\mathrm{ms})\end{array}$ & $\begin{array}{l}\mathrm{W}_{t} \text { of relative speed } \\
\text { algorithm as compared } \\
\text { to dynamic waiting time } \\
\text { algorithm }\end{array}$ \\
\hline V1 & V2 & 100 & 10 & 15 & 0 & 20 & 4.117 & 20 & More \\
\hline V1 & V3 & 100 & 22 & 14 & 0 & 20 & 0.617 & 20 & More \\
\hline V1 & V4 & 100 & 15 & 10 & 0 & 20 & 3.937 & 20 & More \\
\hline V1 & V5 & 100 & 5 & 16 & 0 & 20 & 8.802 & 20 & More \\
\hline V2 & V3 & 100 & 20 & 14 & 0 & 20 & 0.879 & 20 & More \\
\hline V2 & V4 & 100 & 30 & 10 & 0 & 20 & 0.564 & 20 & More \\
\hline V2 & V5 & 100 & 4 & 16 & 0 & 20 & 10.408 & 20 & More \\
\hline V3 & V4 & 100 & 50 & 10 & 0 & 20 & 0.019 & 20 & More \\
\hline V4 & V5 & 100 & 6 & 16 & 0 & 20 & 7.431 & 20 & More \\
\hline V3 & V5 & 100 & 15 & 16 & 0 & 20 & 1.485 & 20 & More \\
\hline
\end{tabular}




\section{CONCLUSION}

To minimize the broadcast storming problem in Vehicular Ad-Hoc Networks efficiently, a relative speed based dynamically broadcasting waiting time algorithm has been proposed for broadcasting the safety/ warning messages in the Vehicular Ad-Hoc Networks. The experiments show that the relative speed of the vehicles has a great concern in broadcast storming problem. Therefore, the proposed relative speed based dynamically broadcasting waiting time algorithm calculates the waiting time for broadcasting the same message again in the network using distance between forwarding and receiving vehicles, broadcasting range of the vehicles and speed of the vehicles relative to each other.

The results of the proposed algorithm has been compared with the results of an already existing dynamically broadcasting algorithm which uses number of neighbors of the receiving vehicle and its distance from the propagating vehicle to calculate the waiting time for rebroadcasting the safety/ warning messages in the network.

The results of both broadcasting algorithms have been compared in different scenarios i.e. in dense networks, in sparse networks and in partially dense and sparse networks. In all scenarios, the results show that the proposed scheme yields more waiting time for broadcasting the messages in the network than already existing waiting time protocols. Particularly, in dense networks, the proposed relative speed based dynamically broadcasting waiting time algorithms performs much better than the already existing dynamically broadcasting waiting time algorithms, distance based algorithms and blind flooding etc.

Due to yielding more waiting time for each vehicle present in dense or sparse network for broadcasting messages in the network as compared to other waiting time protocols, the proposed algorithm is much better in terms of suppression of broadcast storm in the VANETs.

\section{REFERENCES}

[1] Papadimitratos, P., La Fortelle, A., Evenssen, K., Brignolo, R., \& Cosenza, S. Vehicular communication systems: Enabling technologies, applications, and future outlook on intelligent transportation. Communications Magazine, IEEE, 47(11), 84-95, 2009.

[2] Muhlethaler, P., Laouiti, A., \& Toor, Y. Comparison of flooding techniques for safety applications in VANETs. In Telecommunications, 2007. ITST'07. 7th International Conference on ITS (pp. 1-6). IEEE, June, 2007.

[3] Oh, S., Kang, J., \& Gruteser, M. Location-based flooding techniques for vehicular emergency messaging. In Mobile and Ubiquitous SystemsWorkshops, 2006. 3rd Annual International Conference on (pp. 1-9). IEEE, July 2006.

[4] Martinez, F. J., Toh, C. K., Cano, J. C., Calafate, C. T., \& Manzoni, P. A street broadcast reduction scheme (SBR) to mitigate the broadcast storm problem in VANETs. Wireless personal communications, 56(3), 559-572, 2011.

[5] Najafzadeh, S., Ithnin, N., Razak, S. A., \& Karimi, R. Dynamic Broadcasting in Vehicular Ad hoc Networks. International Journal of Computer Theory and Engineering, 5(4), 629-632, 2013.

[6] Suriyapaibonwattana K. and Pomavalai C. “An Effective Safety Alert Broadcast Algorithm for VANET,” IEEE 2008, pp.247-250, 2008.

[7] Chiasserini, C. F., Fasolo, E., Furiato, R., Gaeta, R., Garetto, M., Gribadou, M., \& Zanella, A. Smart broadcast of warning messages in vehicular ad hoc networks. In Workshop Interno Progetto NEWCOM (NoE), November 2005.

[8] Ros, F. J., Ruiz, P. M., \& Stojmenovic, I. Reliable and efficient broadcasting in vehicular ad hoc networks. In Vehicular Technology Conference, 2009. VTC Spring 2009. IEEE 69th (pp. 1-5). IEEE, April, 2009.

[9] Fracchia, R., Meo, M., \& Rossi, D. Avoiding broadcast storms in intervehicular warning delivery services. Proc. of ASWN'06, 2006.

[10] Koubek, M., Rea, S., \& Pesch, D. Event suppression for safety message dissemination in VANETs. In Vehicular Technology Conference (VTC 2010-Spring), 2010 IEEE 71st (pp. 1-5). IEEE, May, 2010.

[11] Iqbal R., Sherazi H.H.R. Comparative Analysis of P-persistence and 1persistence Techniques for Avoiding Broadcast Storming in VANETs. MAGNT Research Report (ISSN.1444-8939) Vol.3(2).PP:407-412, 2015.

[12] Rezaei, F. A Novel Data Dissemination Scheme in Vehicular Networks for Intelligent Transportation System Applications, 2010

[13] Bae, I. H. Design and Evaluation of a Hybrid Intelligent Broadcast Algorithm for Alert Message Dissemination in VANETs. In Advanced Computer Science and Information Technology (pp. 68-77). Springer Berlin Heidelberg, 2011. 